\title{
Age, BMI and diabetes as independent predictors of brain hypoperfusion
}

\author{
Zita Képes ${ }^{1}$ (D), Ferenc Nagy² (D), Ádám Budai² (D), Sándor Barna² (D), Regina Esze ${ }^{3}$ (D), Sándor Somodi3 (iD, Miklós Káplár ${ }^{3}$ (iD, \\ Ildikó Garai1,2 (D), József Varga ${ }^{1}$ iD \\ 'University of Debrecen, Faculty of Medicine, Department of Medical Imaging, Division of Nuclear Medicine and Translational Imaging, \\ Hungary \\ ${ }^{2}$ Scanomed Ltd. Nuclear Medicine Centers, Hungary \\ 3University of Debrecen, Faculty of Medicine, Department of Internal Medicine, Hungary
}

[Received 6 VII 2020; Accepted 6 X 2020]

\begin{abstract}
Background: Cerebral blood flow abnormalities are supposed to be potential risk factors for developing cognitive dysfunction in the general population. Aging, obesity and type 2 diabetes mellitus are associated with perfusion abnormalities leading to cognitive impairment, neurodegeneration and future development of dementia. In our study, we aimed at identifying independent factors that contribute to the appearance of regional brain perfusion changes besides those that are already known.

Material and methods: Forty-three type 2 diabetic and twenty-six obese patients were enrolled. After the intravenous administration of $740 \mathrm{MBq}{ }^{99 \mathrm{mTC}} \mathrm{T}$-hexamethylpropylene amine oxime (HMPAO), all subjects underwent brain perfusion SPECT imaging applying AnyScan S Flex dual-head gamma camera (Mediso, Hungary). Using Philips Achieva 3T scanner brain resting-state functional MRI was also performed. The SPECT and MRI images were co-registered and transformed to the MNI152 atlas space so that data of the following standard volumes of interest (VOIs) could be obtained: frontal lobe, parietal lobe, temporal lobe, occipital lobe, limbic region, cingulate, insula, basal ganglia, cerebrum, limbic system and brain stem. Using the SPSS 25 statistical software package, general linear regression analysis, Student's t-test, and Mann-Whitney U-test were applied for statistical analyses.

Results: Multivariate linear analysis identified that BMI and age are significantly $(p<0.0001)$ associated with perfusion, and patient group was slightly above threshold $(p=0.0524)$. We also found that the presence of diabetes was an independent significant predictor of normalized regional brain perfusion only in the insula $(p<0.001)$. Other independent predictors of normalized regional brain perfusion were: age in the insula $(p<0.001)$ and in the limbic region $(p<0.01)$, and BMI in the brain stem $(p<0.01)$.

Conclusions: Age and BMI proved to be general, and diabetes regional predictor of brain hypoperfusion. BMI appeared to be a novel factor affecting brain perfusion. In one specific region, the insula, we detected a difference between the obese and the diabetic group. These findings may be significant in the understanding of the development of cognitive impairment in metabolic diseases.
\end{abstract}

KEY words: brain perfusion; SPECT; type 2 diabetes mellitus; obesity; insula; limbic region; brain stem

Nucl Med Rev 2021; 24, 1: 11-15

\section{Introduction}

Cerebral blood flow alteration is supposed to be a potential risk factor for developing cognitive dysfunction in the general population [1, 2]. Aging, obesity, and type 2 diabetes mellitus (T2DM) are associated with perfusion abnormalities leading to cognitive

Correspondence to: Zita Képes, University of Debrecen, Faculty of Medicine, Department of Medical Imaging, Division of Nuclear Medicine and Translational Imaging, Hungary e-mail: zitakepes@gmail.com impairment, neurodegeneration and future development of dementia.

Recent data pointed out particular brain areas with perfusion deficit related to aging. Aanerud et al. [3] detected the magnitudes of regional cerebral blood flow (rCBF) and cerebral metabolic rate of oxygen (CMRO2), two significant parameters characterizing brain aging, in areas of cerebral cortex in a PET study with sixty-six healthy volunteers enrolled aged between twenty-one and eighty-one [4]. The inferior parietal lobules showed perfusion deficit in cognitively normal aging subjects by Okonkwo et al. [5]. In a ${ }^{99 m} \mathrm{TC}-\mathrm{HMPAO}$ study with forty healthy young and twenty-eight aged subjects involved, Catafau et al. [6] found significantly lower rCBF ratios in aged subjects than in the young in the left frontal 
lobe and the posterior region of the left temporal lobe. Aging is associated with vascular pathology such as cortical microinfarcts, grey matter lacunae and irreversible endothelial dysfunction [7-13]. These vascular alterations contribute to the appearance of age-related brain hypoperfusion that probably leads to the development of neurodegenerative diseases.

Metabolic conditions such as obesity and T2DM that frequently appear and progress together with aging are also linked to brain perfusion abnormalities that may lead to future neurodegeneration.

According to Bissels et al. [14] the risk of dementia is $73 \%$ higher in type two diabetic patients than in patients without T2DM. The Italian Longitudinal Study on Aging in which patients with mild cognitive impairment and metabolic syndrome were studied supports the connection between obesity and dementia [15]. Birdsill et al. [16] detected a 15 per cent lower mean grey matter cerebral blood flow in subjects with metabolic syndrome that could be associated with decreased memory function. Cui Y. et al. [17] found decreased CBF in T2DM in the posterior cingulate cortex, precuneus and bilateral occipital lobe. Willeumier K. et al. [18] indicated that higher body mass index (BMI) in healthy individuals is associated with decreased rCBF in the following Brodmann areas: $8,9,10,11,32$, and 44 that are brain regions involved in attention, reasoning, and executive function. They also pointed out that an elevated BMI is connected to decreased rCBF in the prefrontal cortex in a group of healthy subjects.

In our study, we aimed at identifying independent factors that contribute to the appearance of regional brain perfusion alterations besides those factors that are already known.

\section{Material and methods}

A total of forty-three type 2 diabetic and twenty-six obese patients were enrolled. The main anthropometric characteristics of the subjects are shown in Table 1. Among the diabetics there were seventeen female and twenty-six male, while in the obese group eighteen female and eight male subjects were involved.

Patients were all recruited from the Department of Internal Medicine of the University of Debrecen as well as a Private General Practice from the city of Miskolc.

Patients were selected based on the following inclusion criteria: age between 18 and 70, manifest obesity (BMI > 30) or controlled type 2 diabetes, and no history of mental or brain disorders. Exclusion criteria included: gravidity, breastfeeding, acute or chronic inflammatory disease, severe liver disease, ongoing steroid treatment, hyperthyroidism, retinoid intake, history of malignant diseases with the exception of basal cell carcinoma, crural ulcer, change in the therapy in the previous 6 months and anticoagulant treatment.

All procedures followed were in accordance with the ethical standards of the responsible national committee on human experimentation (OGYEI/2829-4/2017). Before enrolment, subjects were given detailed information concerning the aims of the study as well as the examinations. Informed consent was collected from all patients involved.

\section{Single-photon emission-computed tomography (SPECT) image acquisition}

AnyScan S Flex (Mediso, Hungary) dual-head gamma-camera equipped with low-energy high resolution parallel hole collimators was used to image brain perfusion. Patients were not prone to stress or heavy emotional burden before the actual examination. Half an hour before the injection of the radiopharmaceutical subjects were given one perchlorate capsule per os to block thyroid uptake. $740 \mathrm{MBq}{ }^{99 m} \mathrm{Tc}$-hexamethylpropylene amine oxime (HMPAO) was administered into the right cubital vein after a ten-minute rest in the dimly-lit examination room.

The main characteristics of SPECT examinations were the following: 120 views, $128 \times 128$ matrix with $2.36 \mathrm{~mm}$ pixel size, $30 \mathrm{sec}$ projection time, with auto body contour.

\section{Brain magnetic resonance imaging (MRI)}

Brain resting-state functional MRI was carried by a Philips Achieva 3T scanner. T1-weighted 3D images (voxel size: $0.5 \times 0.5 \times 1 \mathrm{~mm}$, matrix size: $480 \times 480 \times 175$ ) were obtained. The T1-weighted 3D turbo field echo protocol was applied for image acquisition with 8 ms repetition time (TR) and $3.7 \mathrm{~ms}$ echo time (TE).

\section{Image processing}

First, the ${ }^{99 m}$ TC-HMPAO SPECT image of each subject was registered to his/her T1-weighted MRI image by rigid transformation, using the FMRIB's Linear Image Registration Tool (FLIRT) linear registration software. Then we transformed T1 weighted MRI images of the patients by elastic transformation to the MNI152-space $(2 \times 2 \times$ $2 \mathrm{~mm}$ voxel size) using the advanced normalization tools (ANTS)

Table 1. Most important anthropometric parameters of patients involved

\begin{tabular}{|c|c|c|c|c|c|c|}
\hline & \multicolumn{2}{|c|}{ Obes } & \multicolumn{2}{|c|}{ Diabetes } & \multirow[b]{2}{*}{ test } & \multirow[b]{2}{*}{$\mathbf{P}$} \\
\hline & median & SD & median & SD & & \\
\hline Age & 53.5 & \pm 9.89 & 50 & \pm 8.59 & t & $>0.1$ \\
\hline Height (cm) & 168 & \pm 11.70 & 170 & \pm 8.77 & $\mathrm{t}$ & $>0.1$ \\
\hline Body weight (kg) & 103 & \pm 18.10 & 95 & \pm 21.48 & $\mathrm{t}$ & 0.045 \\
\hline Blood glucose level (mmol/L) & 5.8 & \pm 0.58 & 7.2 & \pm 1.72 & $M-W$ & $<0.0001$ \\
\hline
\end{tabular}

$\mathrm{BMI}$ - body mass index; SD — standard deviation 

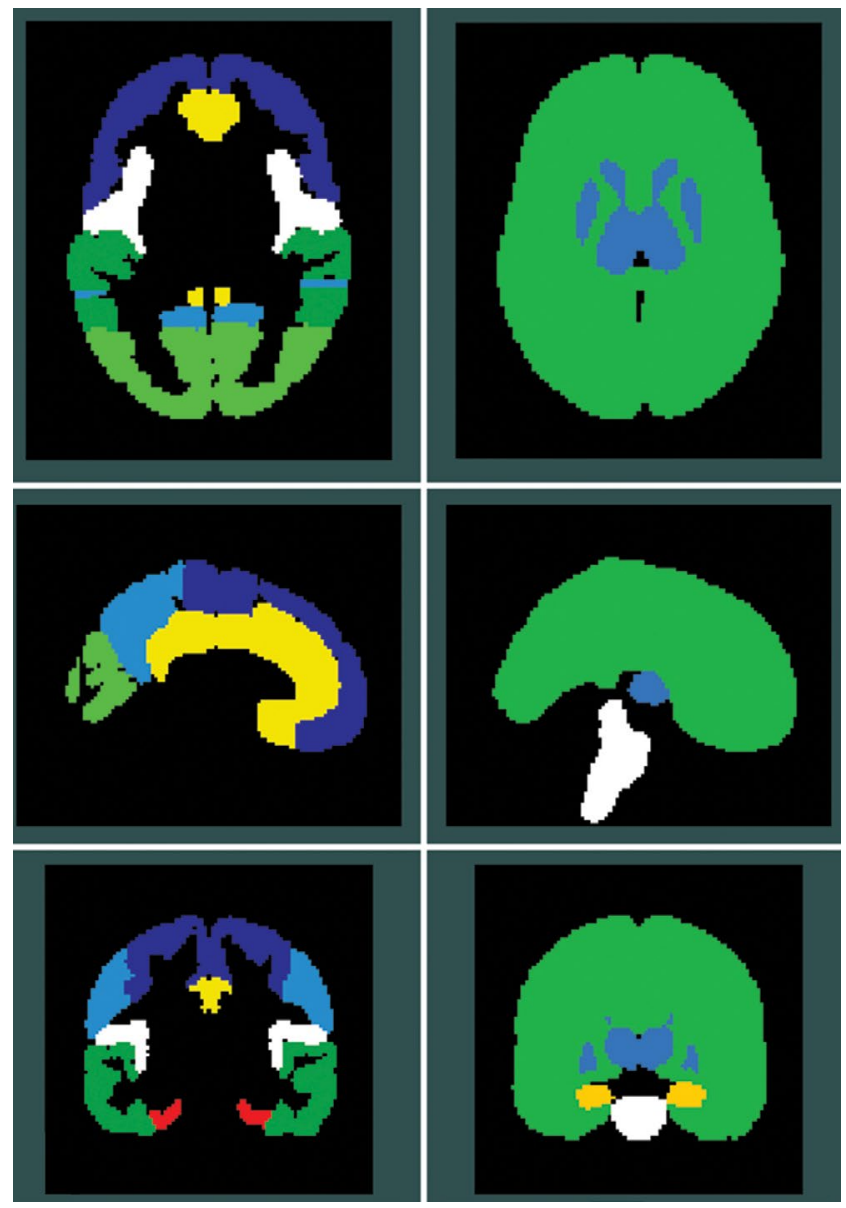

Figure 1. Showing aggregated brain regions created on the basis of Harvard-Oxford atlas linear and non-linear image registration [19]. The combination of both transformations was applied to the SPECT image to transfer it to the MNI space, so that data of the following standard volumes of interest (VOIs) could be obtained: frontal lobe, parietal lobe, temporal lobe, occipital lobe, limbic region, cingulate, insula, basal ganglia, cerebrum, limbic system and brain stem. The aggregated brain regions that were used for VOls are demonstrated in Figure 1.

\section{Statistical analyses}

The SPSS 25 statistical software package (SPSS Inc.) was used for data analysis. To identify influencing factors, general linear regression analysis was performed with Hochberg's correction for multiple regions. Student's t-test was used to compare parameters with normal distribution while Mann-Whitney U test was applied for the comparison of parameters with non-Gaussian distribution.

\section{Results}

We investigated factors that may be associated with cerebral perfusion (normalized to the occipital region) by the general linear model. First, we included the patient group (DM or obese) and gender as factors and age, blood glucose and BMI as covariates. BMI (Mann-Whitney: $p=0.004$ ) and glucose level (Mann-Whitney: $p<0.0001$ ) were integrated in the model since these parameters were significantly different in the two observed groups as seen in Figure 2. However, based on Student's t-test there was no statistically significant difference between the two groups regarding age (Fig. 2), because of previous findings in the literature, we also included age in the analysis. Since the effect of blood glucose level on brain perfusion was far from significant both in the multivariate model and in all regions $(p>0.1)$, we eliminated it from the model.
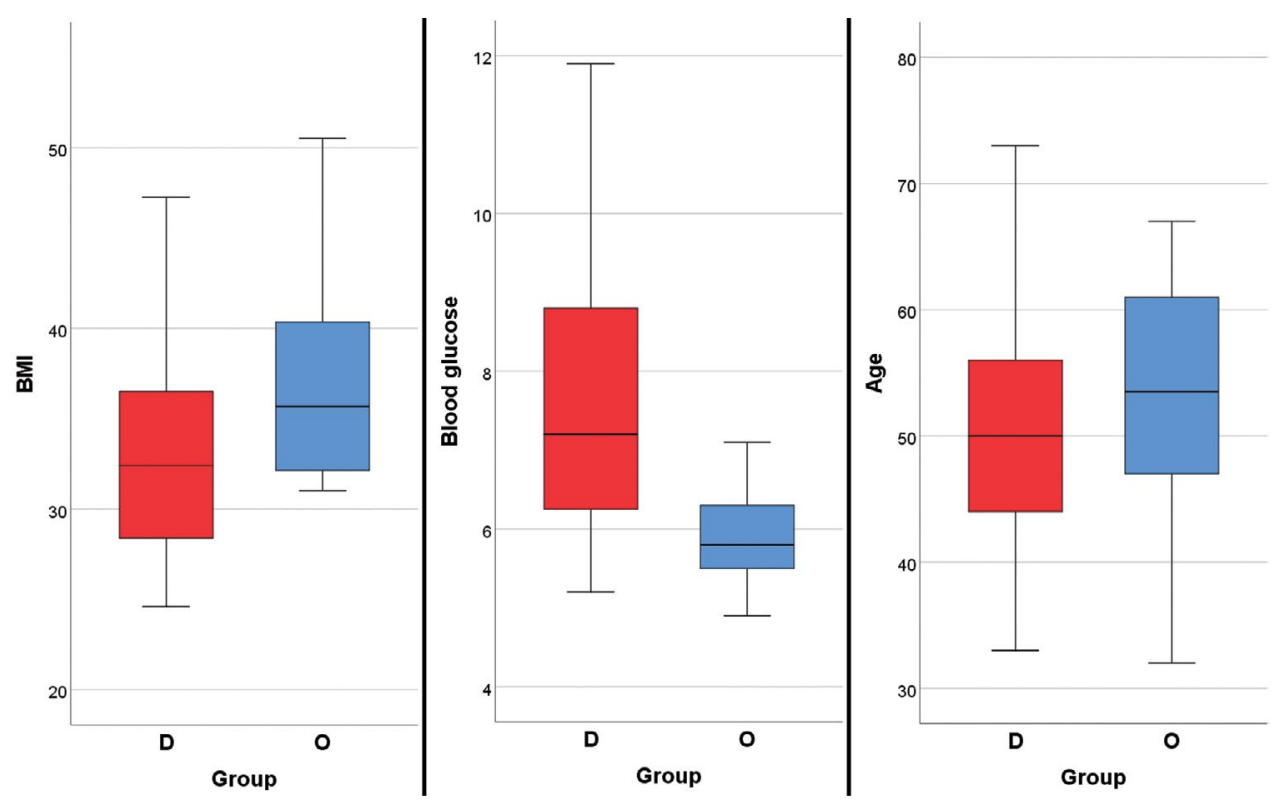

Figure 2. Box-and whiskers plots showing the most important continuous parameters in both groups: BMl, serum blood glucose level and age. $\mathrm{BMI}$ - body mass index 
From the remaining variables, multivariate analysis with all brain regions as dependent variables identified that $\mathrm{BMI}$ and age are significantly ( $p<0.0001$ ) associated with perfusion; and patient group was slightly above threshold $(p=0.0524)$.

Univariate contrast of the group in all but one brain region was negative (normalized perfusion was lower in DM), but after applying Hochberg's correction for multiple comparisons, we found that the presence of DM was an independent significant predictor of normalized regional brain perfusion only in the insula $(p<0.001)$.

Other independent predictors of normalized regional brain perfusion were:

age in the insula $(p<0.001)$ and in the limbic region $(p<0.01)$

$\mathrm{BMI}$ in the brain stem $(p<0.01)$

\section{Discussion}

We detected age-dependent hypoperfusion specifically in the region of the insula and in the limbic region. The direct connection between aging and the involvement of these two specific brain regions is not exactly known.

Age is considered to be an important predictor of hypoperfusion [20]. In the long run cerebral hypoperfusion results in cognitive dysfunction. Bonthius D. J. et al. [21] stated that the limbic region is much more burdened with neurofibrillary tangles than other brain regions. This finding supports the connection between cognitive dysfunction and limbic perfusion abnormality. Since we examined patients with normal cognition we speculate that the early involvement of the limbic region could be characteristic in patients who might be at risk for Alzheimer's disease $(A D)$ even without manifest clinical signs actually present. Since both the insula and the limbic region are related to $A D$ pathology, the involvement of these brain areas in the process of brain aging may predict the appearance of future dementia.

Cerebral perfusion defects induced by aging could be triggered by both obesity and T2DM. We suppose that these metabolic disorders could enhance vascularly triggered age-related cognitive deficit. Our finding is in coherence with the findings of Leanne et al. [22] who detected lower cerebral blood flow in type 2 diabetic patients with mild cognitive impairment in the insula, medial temporal lobes and in the frontal lobes compared to type 2 diabetic patients without mild cognitive impairment and healthy controls. Further, they also reported association between regional cerebral blood flow measurements and Addenbrooke's Cognitive Assessment (ACE-R) score in the insula, thalamus and medial temporal lobes.

Our second finding was BMl-associated hypoperfusion specifically in the brain stem. To our knowledge, there is no similar finding in the literature. The reason behind this result is unclear yet. We presume that an excessive amount of adipose tissue in that brain area possibly has a compressive effect on the vasculature of the brain stem. This may induce and accelerate brain stem hypoperfusion together with high BMI-induced altered insulin regulation, leptin-resistance, inflammatory processes, and vascular abnormalities. Further, obesity-related endothelial dysfunction and disrupted smooth muscle function could be other causes of cerebral hypoperfusion in obesity. All these factors are strongly connected to neurodegeneration. We speculate that BMI-related hypoperfusion in the brain stem may contribute to the development of future dementia. Obesity-induced cerebral blood flow reduction promotes amyloid-beta production that causes endothelial dysfunction leading to cognitive impairment $[22,23]$. This could be in line with the findings of Gunstad J. et al. [25] who detected that people with elevated BMI have reduced executive function. The outcomes of Gustafson et al. [26] also support this hypothesis. They evaluated the connection between BMI and brain atrophy, the main marker of neurodegeneration. They showed that the risk of temporal atrophy increased by $3 \%$ per $1.0 \mathrm{~kg} / \mathrm{m}^{2}$ increase in BMI that could predict the development of cognitive decline.

Finally, we found that the presence of diabetes was an independent significant predictor of normalized regional brain perfusion only in the region of the insula. We speculate that insular perfusion may be affected much earlier by diabetes, and insula is much more vulnerable to metabolic changes than other brain regions. This could be one reason for the unique involvement of disease-related insular perfusion alteration. Although, the fact that we examined well-controlled diabetic patients might also explain why we only detected perfusion difference in the insula. Insula is involved in the pathophysiology of $A D$ [21]. Consequently, our finding puts the emphasis on the elevated risk for dementia in diabetics. Additionally, hyperglycemia characterizing diabetes causes altered endothelial dependent cerebral vasodilation, oversecretion of vasoconstrictor endothelin-1 and blood-brain barrier impairment [27]. These factors could also strengthen the link between hyperglycemia, diabetes and chronic cerebral hypoperfusion which is an important element of vascular cognitive impairment [27].

However, the discrepancy can be detected in the literature concerning the association between diabetes and brain hypoperfusion. Recent research has pointed out that studies involving a small number of patients with severe symptoms tend to detect hypoperfusion in T2DM [28]. In addition, these researchers do not take atrophy into account that could possibly explain hypoperfusion.

There are important limitations to our study worth noting. First, we did not include a healthy control group due to ethical reasons. Second, we involved well-treated diabetic patients taking different types of medications (antidiabetics with different mechanisms of action, antihypertensive and lipid-lowering drugs, and antidiuretics).

\section{Conclusions}

We managed to detect three factors that influence brain perfusion. Age, - and BMl proved to be general, and diabetes regional predictor of brain hypoperfusion. Our result regarding the effect of age on cerebral perfusion that is well known from the literature is in line with previous studies. Based on multiparametric linear analysis BMI appeared to be a relatively new factor influencing brain perfusion. Further, in one specific region, in the insula, we detected difference between the obese and the diabetic group.

Our findings put the emphasis on the multi-aetiological nature of cerebral hypoperfusion. We emphasize that it is important to identify independent factors affecting brain perfusion. This could be essential to help understand the pathological mechanisms that occur behind the elevated risk of developing cognitive impairment in metabolic diseases. 


\section{Acknowledgement}

This research was supported by the National Grant No. GlNOP-2.1.1-15-2015-00609.

\section{Disclosure}

The authors report no biomedical financial interests or potential conflicts of interest.

\section{References}

1. 1Ishiwata A, Sakayori O, Minoshima S, et al. Preclinical evidence of Alzheimer changes in progressive mild cognitive impairment: a qualitative and quantitative SPECT study. Acta Neurologica Scandinavica. 2006; 114(2): 91-96, doi: 10.1111/j.1600-0404.2006.00661.x.

2. Jagust WJ, Eberling JL, Reed BR, et al. Clinical studies of cerebral blood flow in Alzheimer's disease. Ann N Y Acad Sci. 1997; 826: 254-262, doi: 10.1111/j.1749-6632.1997.tb48477.x, indexed in Pubmed: 9329697.

3. Aanerud J, Borghammer P, Chakravarty M, et al. Brain Energy Metabolism and Blood Flow Differences in Healthy Aging. Journal of Cerebral Blood Flow \& Metabolism. 2012; 32(7): 1177-1187, doi: 10.1038/jcbfm.2012.18.

4. Daulatzai MA. Cerebral hypoperfusion and glucose hypometabolism: Key pathophysiological modulators promote neurodegeneration, cognitive impairment, and Alzheimer's disease. J Neurosci Res. 2017; 95(4): 943-972, doi: 10.1002/jnr.23777, indexed in Pubmed: 27350397.

5. Okonkwo OC, Xu G, Oh JM, et al. Cerebral blood flow is diminished in asymptomatic middle-aged adults with maternal history of Alzheimer's disease. Cereb Cortex. 2014; 24(4): 978-988, doi: 10.1093/cercor/bhs381, indexed in Pubmed: 23236200.

6. Catafau A, Lome a F, Pavia J, et al. Regional cerebral blood flow pattern in normal young and aged volunteers: a99mTc-HMPAO SPET study. European Journal of Nuclear Medicine. 1996; 23(10): 1329-1337, doi: 10.1007/bf01367588.

7. Bouras C, Kövari E, Herrmann F, et al. Stereologic Analysis of Microvascular Morphology in the Elderly. Journal of Neuropathology and Experimental Neurology. 2006; 65(3): 235-244, doi: 10.1097/01.jnen.0000203077.53080.2c.

8. Jeynes B, Provias J. The possible role of capillary cerebral amyloid angiopathy in Alzheimer lesion development: a regional comparison. Acta Neuropathol. 2006; 112(4): 417-427, doi: 10.1007/s00401-006-0099-Z, indexed in Pubmed: 16830133.

9. Kövari E, Gold G, Herrmann F, et al. Cortical Microinfarcts and Demyelination Significantly Affect Cognition in Brain Aging. Stroke. 2004; 35(2): 410-414, doi: 10.1161/01.str.0000110791.51378.4e.

10. Kövari E, Gold G, Herrmann FR, et al. Cortical microinfarcts and demyelination affect cognition in cases at high risk for dementia. Neurology. 2007; 68(12): 927-931, doi: 10.1212/01.wnl.0000257094.10655.9a.

11. Gold G, Kövari E, Herrmann F, et al. Cognitive Consequences of Thalamic, Basal Ganglia, and Deep White Matter Lacunes in Brain Aging and Dementia. Stroke. 2005; 36(6): 1184-1188, doi: 10.1161/01.str.0000166052.89772.b5.

12. Thal DR, Capetillo-Zarate E, Larionov S, et al. Capillary cerebral amyloid angiopathy is associated with vessel occlusion and cerebral blood flow disturbances. Neurobiol Aging. 2009; 30(12): 1936-1948, doi: 10.1016/j. neurobiolaging.2008.01.017, indexed in Pubmed: 18359131.

13. Hallam K, Li Q, Ananthakrishnan R, et al. Aldose Reductase and AGE-RAGE pathways: central roles in the pathogenesis of vascular dysfunction in aging rats. Aging Cell. 2010; 9(5): 776-784, doi: 10.1111/j.1474-9726.20 10.00606.x.

14. Biessels $G$, Strachan $M$, Visseren $F$, et al. Dementia and cognitive decline in type 2 diabetes and prediabetic stages: towards targeted interventions. The Lancet Diabetes \& Endocrinology. 2014; 2(3): 246-255, doi: 10.1016/s22138587(13)70088-3.

15. Solfrizzi V, Scafato E, Capurso C, et al. Italian Longitudinal Study on Aging Working Group. Metabolic syndrome, mild cognitive impairment, and progression to dementia. The Italian Longitudinal Study on Aging. Neurobiol Aging. 2011; 32(11): 1932-1941, doi: 10.1016/j.neurobiolaging.2009.12.012, indexed in Pubmed: 20045217.

16. Birdsill AC, Carlsson CM, Willette AA, et al. Low cerebral blood flow is associated with lower memory function in metabolic syndrome. Obesity (Silver Spring). 2013; 21(7): 1313-1320, doi: 10.1002/oby.20170, indexed in Pubmed: 23687103.

17. Cui $Y$, Liang X, Gu H, et al. Cerebral perfusion alterations in type 2 diabetes and its relation to insulin resistance and cognitive dysfunction. Brain Imaging Behav. 2017; 11(5): 1248-1257, doi: 10.1007/s11682-016-9583-9, indexed in Pubmed: 27714551.

18. Willeumier K, Taylor D, Amen D. Elevated BMI Is Associated With Decreased Blood Flow in the Prefrontal Cortex Using SPECT Imaging in Healthy Adults. Obesity. 2012; 19(5): 1095-1097, doi: 10.1038/oby.2011.16.

19. Avants BB, Tustison N, Song G. Advanced normalization tools (ANTS). Insight J. 2009; 2(365): 1-35.

20. Jansen JFA, van Bussel FCG, van de Haar HJ, et al. Cerebral blood flow, blood supply, and cognition in Type 2 Diabetes Mellitus. Sci Rep. 2016; 6(1): 10, doi: 10.1038/s41598-016-0003-6, indexed in Pubmed: 27920431.

21. Bonthius D, Solodkin A, Hoesen GV. Pathology of the Insular Cortex in Alzheimer Disease Depends on Cortical Architecture. Journal of Neuropathology and Experimental Neurology. 2005; 64(10): 910-922, doi: 10.1097/01. jnen.0000182983.87106.d1.

22. HUNT L, SELVARAJAHD, TESFAYE S, et al. Cerebral Blood Flow Abnormalities in Brain Regions Responsible for Cognitive Function in Type 2 Diabetes. Diabetes. 2018; 67(Supplement 1), doi: 10.2337/db18-1798-p.

23. Toda N, Ayajiki K, Okamura T. Obesity-induced cerebral hypoperfusion derived from endothelial dysfunction: one of the risk factors for Alzheimer's disease. Curr Alzheimer Res. 2014; 11(8): 733-744, doi: 10.2174/1567205 01108140910120456, indexed in Pubmed: 25212912.

24. Torre JCd, Pappas BA, Prevot V, et al. Hippocampal nitric oxide upregulation precedes memory loss and A 1-40accumulation after chronic brain hypoperfusion in rats. Neurological Research. 2013; 25(6): 635-641, doi: 10.1179/016164103101201931

25. Gunstad J, Paul RH, Cohen RA, et al. Elevated body mass index is associated with executive dysfunction in otherwise healthy adults. Compr Psychiatry. 2007; 48(1): 57-61, doi: 10.1016/j.comppsych.2006.05.001, indexed in Pubmed: 17145283.

26. Gustafson D, Lissner L, Bengtsson C, et al. A 24-year follow-up of body mass index and cerebral atrophy. Neurology. 2004; 63(10): 1876-1881, doi: 10.1212/01.wnl.0000141850.47773.5f.

27. Livingston JM, McDonald MW, Gagnon T, et al. Influence of metabolic syndrome on cerebral perfusion and cognition. Neurobiol Dis. 2020; 137: 104756, doi: 10.1016/j.nbd.2020.104756, indexed in Pubmed: 31978604.

28. Sabri O, Hellwig D, Schreckenberger M, et al. Influence of diabetes mellitus on regional cerebral glucose metabolism and regional cerebral blood flow. Nucl Med Commun. 2000; 21(1): 19-29, doi: 10.1097/00006231-20000100000005, indexed in Pubmed: 10717898. 